\title{
Interventions to prevent hemodynamic instability during renal replacement therapy for acute kidney injury: a systematic review protocol
}

Adrianna Douvris ${ }^{1}$, Swapnil Hiremath ${ }^{2}$, Lauralyn McIntyre ${ }^{3,4}$, Lindsey Sikora ${ }^{5}$, Catherine Weber ${ }^{6}$ and Edward G. Clark $2,7^{*}$

\begin{abstract}
Background: Hemodynamic instability during renal replacement therapy (HIRRT) in the form of intradialytic hypotension (IDH) is a frequent complication of hemodialysis in end-stage kidney disease (ESKD), and most studies have focused on this chronic population. However, HIRRT is also an important concern for critically ill ICU patients with acute kidney injury (AKI), complicating an estimated 30\% of dialysis treatments in this population. HIRRT can exacerbate organ hypoperfusion in the setting of critical illness and may negatively impact renal recovery in the AKI population. This is a protocol for a systematic review to synthesize the evidence surrounding dialysis-related interventions used to minimize HIRRT in critically ill patients with RRT-requiring AKI. This protocol has been registered with the International Prospective Register of Systematic Reviews (PROSPERO) database.

Methods/design: We will search MEDLINE, EMBASE, and CENTRAL databases in collaboration with a health information specialist using a comprehensive search strategy. We will also supplement our search with a scan of the "gray literature" to identify relevant ongoing trials or conference abstracts. Observational studies and clinical trials will be included in our analysis. Our outcomes will include the incidence of HIRRT, dialysis-related complications, in-hospital mortality, and renal recovery. Prior to our search, we performed an initial search of these databases and PROSPERO, which yielded no prior or ongoing systematic reviews on this topic. Two reviewers will independently screen the list of identified abstracts using pre-defined inclusion and exclusion criteria. Two reviewers will then independently extract data from selected studies and undertake an assessment of their quality using validated tools.

Discussion: HIRRT is a common complication of renal replacement therapy not only in ESKD but also in the critically ill AKI population. It can result in early discontinuation of dialysis, further organ injury from hypoperfusion, and possibly negatively impact renal recovery. This systematic review will synthesize the existing evidence on the interventions employed to predict or prevent episodes HIRRT in critically ill patients with RRT-requiring AKI. This systematic review will allow for an understanding the current evidence for interventions to limit HIRRT in AKI and, in doing so, may also highlight areas in need of further research.
\end{abstract}

Systematic review registration: PROSPERO CRD42016037754

Keywords: Acute kidney injury, Renal replacement therapy, Intradialytic hypotension, Trials, Patients, Blood pressure, Dialysis

\footnotetext{
* Correspondence: edclark@toh.ca

${ }^{2}$ Division of Nephrology, Department of Medicine and Kidney Research

Centre, Ottawa Hospital Research Institute, University of Ottawa, Ottawa,

Canada

${ }^{7}$ The Ottawa Hospital - Riverside Campus, 1967 Riverside Drive, Ottawa,

Ontario K1H 7W9, Canada

Full list of author information is available at the end of the article
} 


\section{Background}

Episodes of hemodynamic instability during renal replacement therapy (HIRRT) in the form of intradialytic hypotension (IDH) frequently complicates maintenance hemodialysis treatments in end-stage kidney disease (ESKD) patients [1]. Minor consequences of this form of HIRRT include symptoms such as postdialysis fatigue $[1,2]$. More serious complications include bowel ischemia, myocardial infarction, and access thrombosis [2]. Recent studies have highlighted that myocardial stunning [3] and brain white-matter changes [4] are also associated with HIRRT in the form of IDH. Furthermore, the occurrence of HIRRT can limit delivery of adequate dialysis treatment as interventions frequently undertaken in response to HIRRT include terminating dialysis sessions early or reducing ultrafiltration goals $[1,2]$. Lastly, in maintenance hemodialysis patients, more frequent episodes of HIRRT (as IDH) may result in more rapid loss of residual renal function over time $[5,6]$.

Most studies of interventions to prevent or limit HIRRT have focused specifically on IDH in maintenance hemodialysis patients with ESKD; however, IDH is only one form of HIRRT which is also an important concern amongst critically ill patients who require hemodialysis or other forms of RRT (such as slow low-efficiency dialysis (SLED) or continuous renal replacement therapy $(\mathrm{CRRT}))$ in the context of acute kidney injury (AKI). AKI that necessitates RRT is a frequent and serious complication of critical illness with an incidence of 2 to $3 \%$ amongst all patients admitted to the intensive care unit (ICU) [7]. One study suggests that HIRRT complicates an estimated $30 \%$ of hemodialysis treatments for AKI patients in the ICU [8]. This is particularly important since there is some evidence to suggest that HIRRT negatively impacts renal recovery in AKI [9], possibly similar to how HIRRT (in the form of IDH) accelerates the loss of residual kidney function in those on maintenance hemodialysis $[5,6]$.

The goal of this study is to summarize all observational and trial evidence related to interventions to prevent or limit HIRRT in AKI patients. This encompasses AKI that is treated with the most commonly utilized RRT modalities in the setting of critical illness (intermittent hemodialysis, SLED, and CRRT).

\section{Objectives}

The primary objective of this systematic review is to synthesize the evidence surrounding interventions to limit HIRRT in critically ill patients with AKI. In particular, we wish to assess the impact of various dialysisrelated interventions that have been studied to minimize its occurrence.

\section{Free form question}

- What is the effectiveness of interventions or strategies employed to limit HIRRT in AKI?

\section{Methods/design}

This protocol is reported in accordance with the PRISMA-P 2015 checklist [10] (see Additional file 1).

\section{Criteria for considering studies for this review}

The planned systematic review and meta-analysis will be performed and reported as proscribed by the Preferred Reporting Items for Systematic Reviews and Meta-Analyses (PRISMA) statement [11]. This protocol was previously registered with the International Prospective Register of Systematic Reviews (PROSPERO): CRD42016037754.

\section{Types of studies}

All studies that assess a dialysis-related intervention to limit or prevent HIRRT in AKI populations, including interventional studies (including cross-over, parallel arm, or cluster trials) and observational (including cohort, cross-sectional, retrospective, prospective, or combined) studies, will be eligible. In addition, to be eligible for inclusion, studies must meet the following criteria.

\section{Population}

Studies with critically ill adult (18 years of age or older) patients with AKI treated with RRT (including intermittent hemodialysis, SLED, or CRRT but excluding those treated with peritoneal dialysis in this context) will be included.

\section{Exposure/intervention}

Studies with a dialysis-related intervention (or a modifiable factor) related to the application of RRT (e.g., dialysate temperature, ultrafiltration method, dialysate electrolyte concentration, sodium profiling, ultrafiltration profiling, medications to prevent or limit HIRRT) will be included. (Observational studies that do not involve an exposure aimed at reducing HIRRT will be excluded).

\section{Comparators}

Only studies that also included critically ill adult patients with AKI treated with RRT that did not receive the exposure or intervention, as a comparitor group, will be included. (Observational studies without an appropriate comparator will also be excluded).

\section{Outcomes}

- Primary outcome: HIRRT. Any definition used is acceptable provided that it is defined by the study's 
methods. HIRRT encompasses not only changes in blood pressure (as in IDH) but also the initiation or increased dosing of vasopressors during RRT and/or any other criteria used to define hemodynamic instability during RRT (such as the need for fluid boluses). Studies that report HIRRT as an outcome but without any definition will be excluded.

- Secondary outcomes: death (any time points reported); ICU and hospital lengths of stay; renal recovery (dialysis independence at any time points reported); increased vasopressor use; need for interventions to treat HIRRT (e.g., fluid bolus, early stop of RRT session, reduction in ultrafiltration goal); cardiovascular events; system clotting; bleeding; dialysis adequacy; treatment-related symptoms (e.g., discomfort/cold/cramping).

\section{Search strategy}

An initial search of MEDLINE and PubMed was performed to assess for prior systematic reviews on this subject. Prospero was searched for any currently registered systematic review on this topic. No prior or ongoing systematic review was identified.

In collaboration with other members of the research team, a health information specialist (LS) has constructed and will implement a comprehensive search strategy (refer to Appendix for sample search strategy).

As our search strategy, we will search the following databases:

- MEDLINE (1950-Feb 2016)

- EMBASE (1980-Feb 2016)

- CENTRAL (Cochrane Central Register of Controlled Trials)

We will supplement our search with a manual scan of bibliographies of all included studies. In addition, a search of the "gray literature" will be performed. This includes a search using "Google Scholar" and a scan of clinical trial registries for ongoing clinical studies (https://www.isrctn.com/). As well, this will include a review of abstracts from past 10 years from relevant scientific meetings:

- American Society of Nephrology Kidney Week

- Society for Critical Care Medicine (included in EMBASE search)

- International Symposium on Intensive Care and Emergency Medicine (included in EMBASE search)

Upon completion, eligible citations will be exported to a citation manager (EndNote, Thompson Reuters Industries, v. X7.3) for screening by two authors (AD, EC).

\section{Study selection}

An initial screen of all identified abstracts will be conducted by two investigators (EC and AD) to determine whether the articles are eligible for further review. The articles considered for further review must have the following requirements:

- Original data from an original study (no review articles or editorials).

- Articles must specifically address HIRRT in patients with AKI.

- Any form of RRT for AKI is eligible except peritoneal dialysis.

- Only studies with titles published in English or French will be considered eligible for inclusion.

- Data from multiple reports assessing the same population or dataset will only be included once. If multiple reports are believed to represent the same population/dataset but report different outcomes or numbers of participants, then clarification will be sought from the corresponding author(s) regarding the totality of reporting for that population/dataset.

The same investigators will then conduct the full-text search to determine whether retrieved articles fulfill inclusion or exclusion criteria. Disagreements will be resolved by consensus where possible and by a third reviewer $(\mathrm{SH})$ if not.

\section{Data extraction}

Prior to duplicate extraction by two independent reviewers $(\mathrm{EC}, \mathrm{AD})$, a data extraction form will be created and piloted. The data extracted will include:

- Study characteristics: first author, year of publication, geographic location, setting (e.g., ICU, post-cardiac surgery), design (number of arms, crossover or not), duration of follow-up

- Sample characteristics: numbers of participants in each arm, age, sex, inclusion and exclusion criteria, baseline imbalances across study arms, other possible confounders

- Details regarding interventions and co-interventions: dose/type of intervention, blinding, dropouts, and study withdrawals

- Outcomes assessed: primary outcome of HIRRT vs other, definition of HIRRT and other outcomes assessed, description of measurement tools/devices.

- Effect of interventions/exposures: data describing categorical or continuous efficacy variables for the included outcomes, adverse events 
Data will be entered into Review Manager (RevMan 5.3) by one reviewer (AD) with verification by the second reviewer (EC).

\section{Quality assessment}

Two independent investigators (EC and AD) will assess the included studies for potential bias and the quality of reporting.

Observational cohort studies will be assessed using the Cochrane Risk of Bias Assessment Tool for NonRandomized Studies of Interventions (ACROBATNRSI) [12]. Quality of reporting for observational studies will be assessed according to the Strengthening the Reporting of Observational Studies in Epidemiology (STROBE) checklist [13]. The Newcastle-Ottawa Scale (NOS) will be implemented to assess the quality of nonrandomized studies with respect to design, content, and ease of use [14]. Any randomized controlled trials (RCTs) will be evaluated using the Cochrane Handbook "Risk of Bias" assessment tool [15].

Any disagreements will be resolved by consensus or, if that is not possible, a third reviewer (SH).

\section{Measures of treatment effect}

RevMan 5.3 will be used for all analyses. For continuous data, we will report the mean differences between groups as well as the $95 \%$ confidence intervals.

\section{Data synthesis}

Data will only be pooled for studies of similar design and limited heterogeneity; however, we expect that significant heterogeneity in interventions, comparator populations, and outcomes will prevent meta-analysis. We plan to examine potential sources of clinical and methodological heterogeneity according to study design, patient population, and outcomes measured as well as intervention and comparator characteristics. Should it be possible to do so, statistical heterogeneity will be assessed using the chi-squared test and defined using the $I^{2}$ statistic with thresholds for interpretation defined in accordance with the Cochrane Handbook for Systematic Reviews of Interventions [15].

Should meta-analysis not be possible or appropriate, the data will be primarily interpreted into a narrative synthesis. This synthesis will be organized according to the intervention/exposure(s) used to attempt to reduce or prevent HIRRT. For each intervention/exposure, they may be subgrouped according to the type of RRT used (i.e., hemodialysis, CRRT, or SLED). Other groupings and subgroupings that may be considered include the following:

- Outcomes of interest

- Cause of AKI
- Indication for RRT

- Treatment setting (e.g., ICU, all hospital, post-operative)

- Methodological quality

- Publication data

- Extent of adjustment for confounding

When possible, data will be interpreted into a quantitative synthesis. Data from included studies will be pooled whenever possible to explore the effect of any identified intervention on the primary or secondary outcomes:

- Frequency of HIRRT

- Mortality

- ICU and hospital length of stay

- Renal recovery

Assessment of reporting biases will be performed using a funnel plot (the standard error of the intervention effect estimate relative to the study size) if there are more than 10 studies included for analysis [16].

\section{Assessing the quality of the evidence}

An assessment of the quality of the evidence for the primary outcome from "very low" to "high" will be made in accordance with the criteria suggested by the Grading of Recommendations Assessment, Development and Evaluation (GRADE) Workgroup [17].

\section{Discussion}

HIRRT is a common complication of renal replacement therapy not only in ESKD but also in the critically ill AKI population. The consequences of HIRRT can range from minor to serious events including bowel ischemia, myocardial infarction, and access thrombosis [2]. HIRRT can lead to further loss of residual renal function over time $[5,6]$. Research suggests that HIRRT can also adversely impact renal recovery in critically ill patients with AKI [9]. To date, most studies on HIRRT and interventions to prevent it are conducted on maintenance hemodialysis patients with ESKD and thus do not necessarily apply to critically ill patients with AKI who require RRT.

This review proposes to use a systematic approach to identify, gather, and summarize the currently existing evidence on interventions to prevent HIRRT in critically ill patients with AKI on RRT from interventional and observational studies as well any ongoing trials. We aim to assess the effectiveness of strategies used to limit or prevent HIRRT in AKI by assessing specific, clinically important outcomes. In addition to exploring strategies to limit HIRRT in AKI, we will also review the available evidence for major outcomes 
associated with HIRRT, which includes mortality, ICU/hospital length of stay, and renal recovery. This summary of evidence can help guide clinical decisions regarding RRT parameters in AKI.

We expect that most of the evidence will come from observational studies rather than RCTs. As there is a higher risk of bias in observational studies, we have chosen to implement in our protocol three accepted assessment tools, the Cochrane ACROBAT-NRSI [10], the STROBE checklist [11], and the Newcastle-Ottawa Scale [12]. We will also use an accepted risk of bias assessment tool for any RCTs [13]. The information we obtain will be dependent on the quality of available evidence, and as such, there will likely be the need for cautious interpretation of the findings. The information from this systematic review will inform clinicians of the current evidence for interventions to limit or prevent HIRRT in AKI and will highlight areas in need of further study. We expect that our summary of evidence will be useful in formulating further research protocols to expand our knowledge in this area, which will ultimately serve to better care for critically ill AKI patients requiring RRT.

\section{Appendix}

Table 1 Sample MEDLINE search strategy

\begin{tabular}{|c|c|}
\hline 1 & exp Acute Kidney Injury/ \\
\hline 2 & (acute adj (kidney or renal or nephr* or tubular or dialys*)).ti. \\
\hline 3 & (acute adj2 (kidney or renal)).tw. \\
\hline 4 & $\begin{array}{l}\left(\left(c_{e} \text { scent }{ }^{*} \text { or progressive or anca* or acute) and (glomerul* }\right.\right. \\
\text { or nephrit*)).ti. }\end{array}$ \\
\hline 5 & ((kidney or renal) adj isch?emi*).ti. \\
\hline 6 & *Nephritis, Interstitial/ci [Chemically Induced] \\
\hline 7 & *Hemorrhagic Fever with Renal Syndrome/ \\
\hline 8 & (induced adj (kidney injury or renal injury)).tw. \\
\hline 9 & Oliguria/ \\
\hline 10 & acute nephr*.tw. \\
\hline 11 & (pre-renal or prerenal).tw. \\
\hline 12 & ((arf or aki) and (renal or kidney)).tw. \\
\hline 13 & $\begin{array}{l}\left.\left(\left(\text { nephropath* and (contrast* adj (medi* or induced or agent* }{ }^{*}\right)\right)\right) \\
\text { or radiocontrast* or iodinated or crystal* or cast).mp. }\end{array}$ \\
\hline 14 & $($ nephrotox* or $($ renal and toxi*)).ti. \\
\hline 15 & renal tubul*.ti. \\
\hline 16 & 14 or 15 \\
\hline 17 & ci.fs. or contrast medi*.tw. or induced.mp. \\
\hline 18 & 16 and 17 \\
\hline 19 & ((kidney or renal) adj isch?emi*).tw. \\
\hline 20 & Kidney Tubules, Proximal/ \\
\hline 21 & ur?emi*.ti. \\
\hline
\end{tabular}

Table 1 Sample MEDLINE search strategy (Continued)

\begin{tabular}{|c|c|}
\hline 22 & renal inflammation.tw. \\
\hline 23 & 19 or 20 or 21 or 22 \\
\hline 24 & *Reperfusion Injury/ \\
\hline 25 & isch?emi* reperfusion.tw. or injury.ti. or acute.tw. \\
\hline 26 & 24 or 25 \\
\hline 27 & 23 and 26 \\
\hline 28 & h?emolytic ur?emi*.ti. \\
\hline 29 & (thrombotic adj (thrombocytopeni* or microangiopathy)).tw. \\
\hline 30 & 28 or 29 \\
\hline 31 & (kidney or renal or acute).mp. \\
\hline 32 & 30 and 31 \\
\hline 33 & (induced adj (kidney or renal)).tw. \\
\hline 34 & (nephrotox* or contrast medi* or reperfusion or perfusion).mp. \\
\hline 35 & 33 and 34 \\
\hline 36 & $\begin{array}{l}\text { ((tubulointerstitial or interstitial or anti-glomerular or } \\
\text { antiglomerular) and (glomerul* or nephrit*)).ti. }\end{array}$ \\
\hline 37 & (acute or crescentic or atypical or progressive).mp. \\
\hline 38 & 36 and 37 \\
\hline 39 & $\begin{array}{l}1 \text { or } 2 \text { or } 3 \text { or } 4 \text { or } 5 \text { or } 6 \text { or } 7 \text { or } 8 \text { or } 9 \text { or } 10 \text { or } 11 \text { or } 12 \text { or } \\
13 \text { or } 18 \text { or } 27 \text { or } 32 \text { or } 35 \text { or } 38\end{array}$ \\
\hline 40 & exp Renal Replacement Therapy/ \\
\hline 41 & ((kidney* or renal) adj3 replacement adj2 therap*).tw. \\
\hline 42 & exp Renal Dialysis/ \\
\hline 43 & ((renal or extracorporeal) adj2 dialys\#s).tw. \\
\hline 44 & exp Hemofiltration/ \\
\hline 45 & h?mofiltration*.tw. \\
\hline 46 & h?modialys\#s.tw. \\
\hline 47 & h?modialfiltration*.tw. \\
\hline 48 & Dialysis/ \\
\hline 49 & cavf.tw. \\
\hline 50 & sustained low-efficiency dialysis.tw. \\
\hline 51 & sled.tw. \\
\hline 52 & or/40-51 \\
\hline 53 & hypotension/ or hypotension, orthostatic/ \\
\hline 54 & hypotens*.tw. \\
\hline 55 & low blood pressure.tw. \\
\hline 56 & vasopressor*.tw. \\
\hline 57 & IDH.tw. \\
\hline 58 & h?modynamic. tw. \\
\hline 59 & or/53-58 \\
\hline 60 & 39 and 52 and 59 \\
\hline 61 & exp Adult/ \\
\hline 62 & adult.tw. \\
\hline 63 & 61 or 62 \\
\hline 64 & 60 and 63 \\
\hline
\end{tabular}




\section{Additional file}

Additional file 1: PRISMA-P 2015 Checklist. (DOCX 36 kb)

\begin{abstract}
Abbreviations
ACROBAT-NRSI: Assessment Tool for Non-Randomized Studies of Interventions; AKI: Acute kidney injury; CRRT: Continuous renal replacement therapy; ESKD: End-stage kidney disease; GRADE: Grading of Recommendations Assessment, Development and Evaluation; HIRRT: Hemodynamic instability during renal replacement therapy; ICU: Intensive care unit; IDH: Intradialytic hypotension; NOS: Newcastle-Ottawa Scale; PRISMA: Preferred Reporting Items for Systematic Reviews and Meta-Analyses; PROSPERO: Prospective Register of Systematic Reviews; RCT: Randomized controlled trial; RRT: Renal replacement therapy; SLED: Slow low-efficiency dialysis; STROBE: Strengthening the Reporting of Observational Studies in Epidemiology
\end{abstract}

\section{Acknowledgements}

Not applicable.

\section{Funding}

No funding was received for this review protocol.

\section{Availability of data and materials}

Not applicable.

\section{Authors' contributions}

EC conceived of the study. AD and LS carried out the initial background research. $E C$ and $A D$ drafted the manuscript. $A D, E C$, and $L S$ drafted the proposed search strategy. SH, LM, LS, and CW made substantial contributions to the design of the project and helped in drafting the manuscript or revising it critically for important intellectual content. EC is the guarantor of the manuscript. All authors gave final approval of the submitted manuscript.

\section{Authors' information}

Not applicable.

\section{Competing interests}

The authors declare that they have no competing interests.

\section{Consent for publication}

Not applicable.

\section{Ethics approval and consent to participate}

Not applicable.

\section{Publisher's Note}

Springer Nature remains neutral with regard to jurisdictional claims in published maps and institutional affiliations

\section{Author details}

'Department of Medicine, University of Ottawa, Ottawa, Ontario, Canada. ${ }^{2}$ Division of Nephrology, Department of Medicine and Kidney Research Centre, Ottawa Hospital Research Institute, University of Ottawa, Ottawa, Canada. ${ }^{3}$ Division of Critical Care, Department of Medicine, The Ottawa Hospital, Ottawa, Ontario, Canada. ${ }^{4}$ Centre for Transfusion Research, Clinical Epidemiology Program, Ottawa Hospital Research Institute, Ottawa, Ontario Canada. ${ }^{5}$ Health Sciences Library, University of Ottawa, Ottawa, Ontario, Canada. 'Division of Nephrology, McGill University Health Centre, Montreal, Quebec, Canada. ${ }^{7}$ The Ottawa Hospital - Riverside Campus, 1967 Riverside Drive, Ottawa, Ontario K1H 7W9, Canada.

Received: 1 July 2016 Accepted: 7 June 2017

Published online: 15 June 2017

\section{References}

1. Assimon MM, Flythe J. Intradialytic blood pressure abnormalities: the highs, the lows and all that lies between. Am J Nephrol. 2015;42:337-50.

2. RF R. Attending rounds: a patient with intradialytic hypotension. Clin J Am Soc Nephrol. 2014;9:798-803.
3. Burton JO, et al. Hemodialysis-induced cardiac injury: determinants and associated outcomes. Clin J Am Soc Nephrol. 2009:4(5):914-20.

4. Eldehni MT, Odudu A, Mclntyre CW. Randomized clinical trial of dialysate cooling and effects on brain white matter. J Am Soc Nephrol. 2015;26(4):957-65.

5. JT D. Pathophysiology of dialysis hypotension: an update. Am J Kidney Dis. 2001;38:S11-7.

6. Misra M, Vonesh E, Van Stone JC, Moore HL, Prowant B, Nolph KD. Effect of cause and time of dropout on the residual GFR: a comparative analysis of the decline of GFR on dialysis. Kidney Int. 2001;59:754-63.

7. Wald $\mathrm{R}$, et al. Changing incidence and outcomes following dialysis-requiring acute kidney injury among critically ill adults: a population-based cohort study. Am J Kidney Dis. 2015;65(6):870-7.

8. Tonelli M, Astephen P, Andreou P, Beed S, Lundrigan P, Jindal K. Blood volume monitoring in intermitent hemodialysis for acute renal failure. Kidney Int. 2002;62:1075-80.

9. Manns M, Sigler MH, Teehan BP. Intradialytic renal haemodynamics potential consequences for the management of the patient with acute renal failure. Nephrol Dial Transplant. 1997;12:870-2.

10. Moher D, et al. Preferred Reporting Items for Systematic Review and MetaAnalysis Protocols (PRISMA-P) 2015 statement. Syst Rev. 2015;4:1.

11. Liberati $A$, et al. The PRISMA statement for reporting systematic reviews and meta-analyses of studies that evaluate healthcare interventions: explanation and elaboration. BMJ. 2009:339:b2700.

12. Sterne JAC, Higgins JPT, Reeves BC. A Cochrane risk of bias assessment tool: for non-randomized studies of interventions (ACROBAT-NRSI). Version 1.0.0. 2014. Available from https://sites.google.com/site/riskofbiastool/. Accessed 11 June 2017.

13. von Elm E, et al. The Strengthening the Reporting of Observational Studies in Epidemiology (STROBE) statement: guidelines for reporting observational studies. J Clin Epidemiol. 2008:61(4):344-9.

14. Wells GA, S.B., O'Connell D, Peterson J, Welch V, Losos M, Tugwell P, Newcastle-Ottawa quality assessment scale case control studies. Available at: http://www.ohri.ca/programs/clinical_epidemiology/nosgen.pdf. Accessed 11 June 2017.

15. Higgins JP, et al. The Cochrane Collaboration's tool for assessing risk of bias in randomised trials. BMJ. 2011:343:d5928.

16. Sterne JA, et al. Recommendations for examining and interpreting funnel plot asymmetry in meta-analyses of randomised controlled trials. BMJ. 2011:343:d4002.

17. Guyatt $\mathrm{GH}$, et al. GRADE: an emerging consensus on rating quality of evidence and strength of recommendations. BMJ. 2008:336(7650):924-6.
Submit your next manuscript to BioMed Central and we will help you at every step:

- We accept pre-submission inquiries

- Our selector tool helps you to find the most relevant journal

- We provide round the clock customer support

- Convenient online submission

- Thorough peer review

- Inclusion in PubMed and all major indexing services

- Maximum visibility for your research

Submit your manuscript at www.biomedcentral.com/submit
BioMed Central 